\title{
Role de la tomodensitométrie du massif facial dans la voie d'abord des sinus maxillaires pour l'extraction des corps étrangers d'origine dentaire
}

\author{
Vidaud C, Tallandier E, Lascar B, Nguyen T, Poidatz E \\ Service d'Odontologie, Hôpital Louis Mourier, Université René Descartes, Paris, France \\ chirdent@hotmail.com
}

L'embryologie permet de comprendre les relations pathologiques entre dents et sinus maxillaires. Le chirurgien dentiste peut être confronté à différentes situations cliniques : soit une dent ectopique évoluant dans le sinus, soit un corps étranger d'origine dentaire expulsé dans le sinus après une manipulation iatrogène. Dans ces deux configurations, une pathologie sinusienne peut apparaitre. Il est alors nécessaire d'aller chercher le corps étranger dans le sinus et de l'éliminer.

Deux voies d'abord chirurgicales permettent d'aborder les sinus maxillaires: la voie endonasale (abord du sinus par les méats nasaux inférieur mais surtout moyen) et la voie externe (Cadwell Luc plus ou moins modifiée).

Les variantes anatomiques des cavités naso-sinusiennes de la face sont fréquentes : il a été dénombré une quinzaine de variations majeures (jusqu'à $62 \%$ de la population est porteuse d'une déviation du septum nasal). Elles peuvent être source de complications iatrogènes lors des explorations endovasculaires ou de la chirurgie endo-nasale (lésions vasculaire, nerveuse ou osseuse)

La tomodensitométrie du massif facial permet un bilan anatomique précis préopératoire qui recherche les variantes anatomiques exposant à un risque de complications de la chirurgie endonasale, et seul cet examen permet une étude complète.

La chirurgie endonasale vidéo-endoscopique est réalisée le plus souvent en première intention car la voie d'abord externe peut présenter de nombreuses complications (Low 1995).

Toutefois, certaines situations peuvent constituer un frein à l'utilisation de cette voie d'abord obligeant parfois à avoir recours à la voie d'abord externe. À ce titre et afin de limiter le recours à la voie de Cadwell-Luc modifiée, un bilan préopératoire précis par tomodensitométrie doit être réalisé. Il apprécie la localisation et la taille du corps étranger d'origine dentaire et les anomalies anatomiques, et indique une voie d'abord externe (Lehmann et al. 2008). 